\title{
Fractal Analysis of Trunk Bark in Relation to Age of Trees: Case Study in Plum
}

\author{
Florin SALA ${ }^{\left.1^{*}\right)}$, Marius BOLDEA ${ }^{2)}$ \\ ${ }^{1)}$ Soil Science and Plant Nutrition, Banat University of Agricultural Sciences and Veterinary Medicine \\ "Regele Mihai I al României" Timisoara, Calea Aradului 119, Romania. \\ ${ }^{2)}$ Mathematics and Statistics, Banat University of Agricultural Sciences and Veterinary Medicine \\ "Regele Mihai I al României” Timisoara, Calea Aradului 119, Romania. \\ ${ }^{*}$ Coresponding author, e-mail: florin_sala@usab-tm.ro
}

Bulletin UASVM Horticulture 71(2) / 2014

Print ISSN 1843-5254, Electronic ISSN 1843-5394

DOI:10.15835/buasvmcn-hort:10299

\begin{abstract}
The fractal geometry of bodies' represents genuine bar codes of the fractal type which, once deciphered, offer stable and trustworthy information on the status and characteristics of the respective bodies. Bark Fractal Tree Age (BFTA) Method presents a non-destructive alternative for the evaluation of the age of trees based on the fractal characteristics of the geometry of trunk bark. Fractal analysis was performed for the trunk bark of plums, set in age categories between 10 and 50 years. Negative correlation was identified between the fractal dimensions (D) of trunk bark geometry and the age of trees, with statistical certainty $\left(R^{2}=-0.856 ; p<0.01\right)$. The variation coefficient $(\mathrm{CV})$ indicated variable dynamics of fractal dimensions in relation to the age of trees, with high values in the case of young trees ( $\mathrm{CV}=0.256$ to 0.502 for age groups between 10 and 20 years) and lower values for old trees (CV = 0.165 to 0.210 for age groups from 40 to 50 years). The BFTA Method was used for obtaining a prediction model for the age of trees based on fractal dimensions, with high statistical certainty $\left(p<0.01 ; R^{2}=0.738\right.$ for the age group from 10 to 40 years, RMSE $=0.882$ to 7.046 and $R^{2}=0.971$ for a shorter interval, namely 20 to 40 years, RMSE $=$ 0.387 to 1.636). Fractal analysis is a tool for developing non-destructive methods for analyzing the dynamics of trunk geometry and ages of trees.
\end{abstract}

Keywords: bark, BFTA Method, fractal geometry, plum age, trunk

\section{INTRODUCTION}

Fractal theory facilitates the qualitative and quantitative description of the irregularities inherent to the geometry of any phenomenon or object, giving the possibility of approaching complexity at various scales. Fractal analysis brings a qualitative and quantitative measure to structures that are limitlessly complex, making it easy to change the scale at which the analysis is made at. From this perspective, biological structures present fractal properties and can be considered as approximate fractals (Mandelbrot, 1983).

Based on studies and research, Bour and Davy (1997) and Bour et al. (2002) considered that some structures do not imply self-similarity or fractality. Certain phenomena or geometric structures present fractal behavior in a limited interval scale, as compared to mathematical fractals (Mandelbrot, 1983; Thorarinsson and Magnusson, 1990; Turcotte, 1992; Cortini and Barton, 1994; Hein, 1999).

Due to the perspectives offered by fractal theory, the interest for using fractal analysis in various fields of research (architecture, biology, chemistry, physics, engineering, etc) has known major increase in recent years. Fractal analysis has the capacity to analyze irregular geometries or fragmented shapes of some big and complex structures which cannot be described with the help of traditional, Euclidian geometry. A number of studies have used fractal analysis to study the different textures and shapes (Backes and Bruno, 2010; Culbert etal., 2012; Xu etal., 2012; Backes and 
Bruno, 2013; Florindo and Bruno, 2013; Gonçalves and Bruno, 2013; Zhao et al., 2013). Fractal analysis has also been used in the characterization of plant species based on measurable fractal properties as fractal dimensions (Bruno et al., 2008; Cope et al., 2012; Du et al., 2013).

Together with leaves, flowers and fruit, trunk bark is an element used in the characterization and identification of trees. Some studies analyzed trunk bark in order to characterize and identify certain species with the help of imaging and fractal analysis (Huang et al., 2006; Fiel and Sablatnig, 2011; Sulc and Matas, 2013). MacFarlane et al. (2014) used non-destructive methods for calibrating tree biomass equations derived from branching architecture based on fractal geometry. Othmani et al. (2014) used 3D technology in bark analysis for the identification and classification of tree species.

There is no mention in specialized literature of fractal analysis being used in the study of trunk bark in order to find the ages of trees. Starting from general considerations on fractal properties of the biological world and the importance of bark geometry in tree characterization, the present research aimed at using bark fractal geometry analysis for characterizing plum trees of various ages.

\section{MATERIALS AND METHODS}

The aim of this study was to assess the fractal characteristics of trunk bark of plums and to correlate them with the ages of trees, in order to develop a method for analyzing and predicting the age of trees based on numerically quantifiable fractal properties, fractal dimension (D).

The biological material used was represented by Gras plum variety with relatively high ecological plasticity and good quality indexes. The trees were in a classic exploitation system, with no technological influence on the morpho-anatomical structure of their trunk. The trunk area analyzed was $1 \mathrm{~m}$ above ground.

The pictures were taken with high-resolution digital camera: Nikon D800E, 36MP, lens Sigma $35 \mathrm{~mm}$ f1.4. The conditions for image capture were unitary, F-stop f/5, Exposer time 1/60s, ISO 250, focal length $35 \mathrm{~mm}$. High-resolution images were obtained, 24 bit depth of field, jpg format. Equal areas (6000x1500 pixels) were extracted from the digital images, for assuring uniformity in the fractal analysis.
The fractal analysis was made using the BoxCouting method, as this is considered to be the most commonly used method for determining fractal dimension (D) (Liebovitch and Toth, 1989; Hou et al., 1990). Twelve partial samples were analyzed for each tree age group; thus, the total number of determinations was carried out 84 . The accuracy of the fractal analysis was high $\left(\mathrm{R}^{2}\right.$ for $\mathrm{D}$ $=0.998-0.999$ ). Fractal spectra were determined with HarFA software, which was developed at the Institute of Physical and Applied Chemistry, Technical University of Brno in the Czech Republic (Buchníček et al., 2000; Nežádal et al., 2001; Zmeškal et al., 2001).

Based on the intermediary values of fractal dimensions (D) resulted from the analysis of partial samples, relation (1) the mean fractal values were calculated, relation (3).

$$
D=m\left[\frac{\ln (\mathbf{F})}{\ln (\varepsilon)}\right]
$$

where: $\quad D$ - fractal dimension; $m$ - slope to regression line, equation (2);

$\mathrm{F}$ - number of new part; $\varepsilon$-scale applied to an object.

$m=\frac{n \sum S C-\sum S \sum C}{n \sum S^{2}-\left(\sum S\right)^{2}}$

where: $\quad m$-slope of the regression line;

$S$ - log of scale or size;

$C$ - log of count;

$n$ - number of size;

$$
\text { Mean } D=\sum \frac{D}{G R I D S}
$$

Within the fractal analysis, for higher certainty of results, the Standard Error for regression line (SE) was calculated, relation (4).

$$
S E=\sqrt{\frac{\sum C^{2}-b \sum C-m \sum S C}{n-2}}
$$

where: $\quad S=\log$ of scale or size;

$C=\log$ of count;

$n=$ number of size; 
$b=\mathrm{y}$-intercept of the regression line;

$m=$ slope of the regression line.

Correlation $\mathrm{R}^{2}$ for the regression line showed the relationship between the log of count and size, it being calculated with relation (5):

$R^{2}=\left[\frac{n \sum S C-\sum S \sum C}{\sqrt{\left(n \sum S^{2}-\left(\sum \llbracket S\right)^{2}\right)\left(n \sum C^{2}-\left(\sum C\right)^{2}\right)}}\right]^{2}(5)$

where: $\quad S=\log$ of scale or size;

$C=\log$ of count;

$n=$ number of size;

The statistical analysis of the experimental data was made with the statistic module of EXCEL in the Office 2007 package and in the statistical and mathematical module of PAST software (Hammer et al., 2001).

\section{RESULTS AND DISCUSSIONS}

The digital images were analyzed in view of evaluating the fractal properties of plum bark geometry. Through fractal analysis over the entire variation interval of the digital information (pixels ratio between $0-255$ ), the fractal spectra were obtained for each age category under analysis, Figure 1.

On the binary images (Figure 1, b indices), BoxCounting analysis allowed the fractal dimensions (D), presented in Table 1. For each fractal dimension, the correlation level was calculated for $\mathrm{D}\left(\mathrm{R}^{2}\right)$ and standard error (SE), Table 1.

Fractal dimensions (D) presented wide variation between $1.936 \pm 0.056$ in the 10 -year age group and 1.852 \pm 0.036 in the 40-year age group.

Based on ANOVA single factor statistical analysis, overall the experimental results presented high statistical certainty $(\mathrm{p}<<0.001$; $\mathrm{F}_{\text {theoretical }}<\mathrm{F}_{\text {calculated }}$, for Alfa $=0.001$ ), Table 2 .

While the bark of young trees is smooth, the bark of older trees becomes rough and exfoliated, bearing the name of rhytidome. The complex geometry of rhytidome (Whitmore, 1962), given by its structure, color and type of exfoliation, is different among tree species and varieties, and it constitutes a key to identification (Chi et al., 2006).

Tab. 1. Variation of the values of fractal dimensions (D) in relation to the age of trees in the case of plum, Gras variety

\begin{tabular}{ccc}
\hline $\begin{array}{c}\text { Age group of trees } \\
\text { (years) }\end{array}$ & $\begin{array}{c}\text { Fractal dimension } \\
\text { (D) }\end{array}$ & $\mathrm{R}^{2}$ for $\mathrm{D}$ \\
\hline 10 & $1.936 \pm 0.056$ & 0.998 \\
\hline 20 & $1.949 \pm 0.064$ & 0.998 \\
\hline 25 & $1.926 \pm 0.053$ & 0.999 \\
\hline 30 & $1.916 \pm 0.054$ & 0.999 \\
\hline 35 & $1.885 \pm 0.049$ & 0.999 \\
\hline 40 & $1.852 \pm 0.036$ & 0.999 \\
\hline 50 & $1.894 \pm 0.052$ & 0.999 \\
\hline
\end{tabular}

Tab. 2. ANOVA single factor statistical analysis for highlighting the source of variation and certainty of results

\begin{tabular}{ccccccc}
\hline Source of Variation & SS & df & MS & F & P-value & F crit \\
\hline Between Groups & 0.081937 & 6 & 0.013656 & 502.6469 & $1.36 \mathrm{E}-59$ & 4.223529 \\
\hline Within Groups & 0.002092 & 77 & $2.72 \mathrm{E}-05$ & & & \\
\hline Total & 0.084029 & 83 & & & & \\
\hline
\end{tabular}

Alfa $=0.001$ 


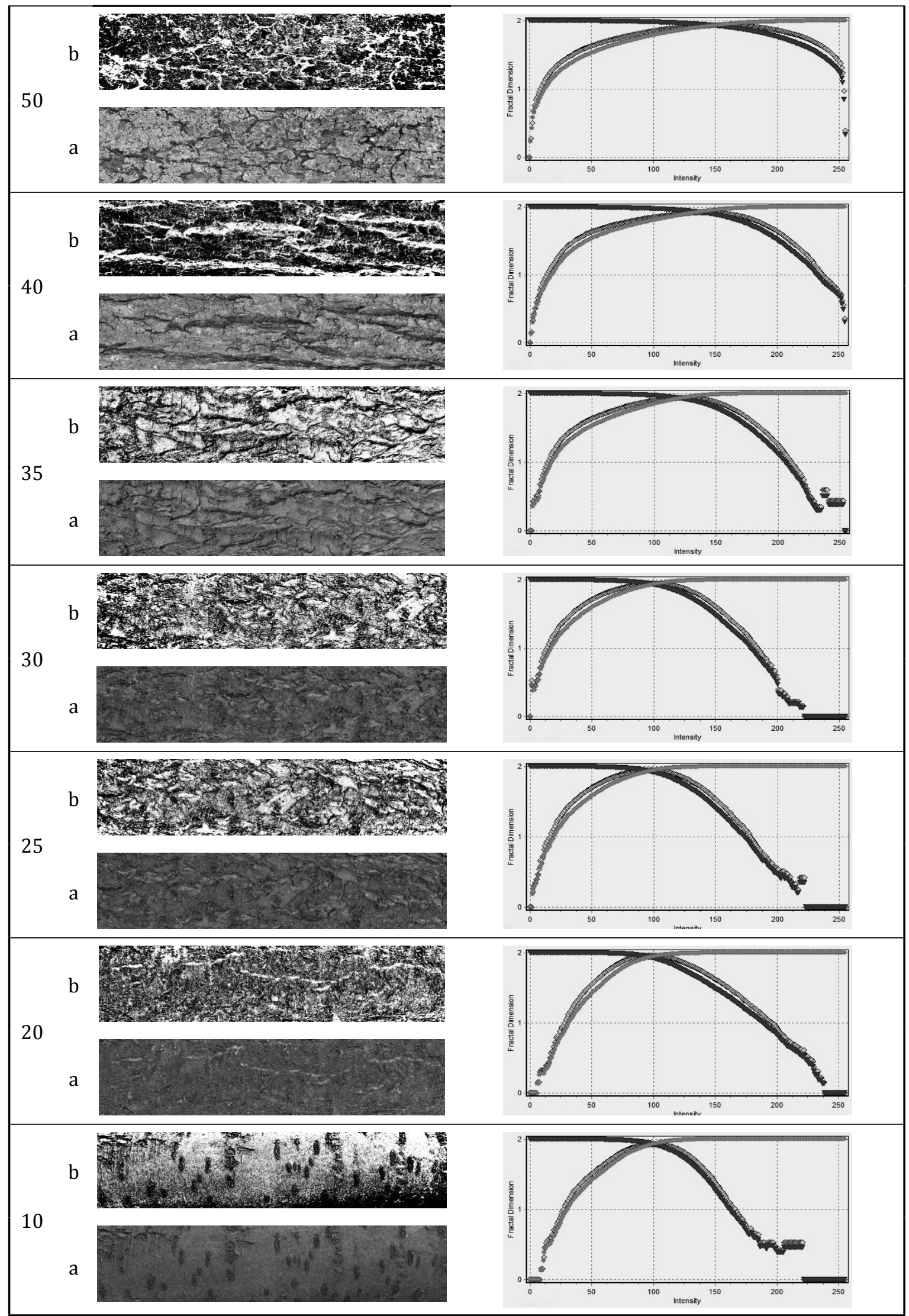

Fig. 1. Images of the plum trunks under study: 10 - 50 age groups of plums; a -original images, b - binary images; fractal spectrum on age groups, to the right side of the image 
Following the variable geometry of bark, fractal properties revealed and expressed this dynamics, with different values of the variation coefficient in different age groups under analysis. In the present study, the bark of 10-year-old trees was smoother, with high elasticity and with specific formations. Changes in the aspect of the bark and in bark geometry of young trees make up a dynamic process, given by the increase in trunk diameter. Certain elements on the bark surface, specific for the growth process and for the expansion of the trunk area, determined specific fractal values.

On the bark of older trees (age group 25 to 35 years old), certain lignified neo-formations, rhytidome, with specific and stable geometric structure. These elements generated variation in bark geometry, which in turn determined other fractal values, specific for these age categories.

The fractal dimensions' variation coefficient revealed the dynamics of the geometry of the morpho-anatomical structure of tree trunks in relation to age groups. The variation coefficient had a wider amplitude with young trees, group 10 to 20 years old (CV $=0.256-0.502$ ), which described the accelerated dynamics of changes in the geometric structure of the bark of young trees. In older trees ( 40 to 50 years old), the variation coefficient recorded was smaller $(\mathrm{CV}=0.165-$
0.210 ), because of the stability of the geometrical structure of the rhytidome, which is a permanent morpho-anatomical structure of the trunk.

The relation between the fractal dimension (D) and the age of trees was described as a second degree polynomial function, equation (6), with high degree of statistical certainty $\left(\mathrm{p}<0.001 ; \mathrm{R}^{2}\right.$ $=-0.856$ ), for the confidence limit of 0.95 . This behavior was recorded in the age interval from 10 to 40 years old. In the case of the 50 -year-old trees under study, the presence of lichens on the trunk determined changes in the geometric structure of the bark, which brought about deviations from this model. The particular distribution of the fractal dimensions in relation to the age of the trees is represented in Figure 2.

$$
y=-0.0002 x^{2}+0.0057 x+1.8992
$$

where: $\quad y=$ fractal dimension (D); $\mathrm{x}=$ age of trees (year);

$a_{0}, a_{1}, a_{2}-$ coefficients given by the experimental conditions.

Based on the correlation between fractal dimensions (D) and the age of trees, Bark Fractal Tree Age (BFTA) Method, Figure 3, was proposed to assess the age of trees based on the fractal properties of trunk bark.

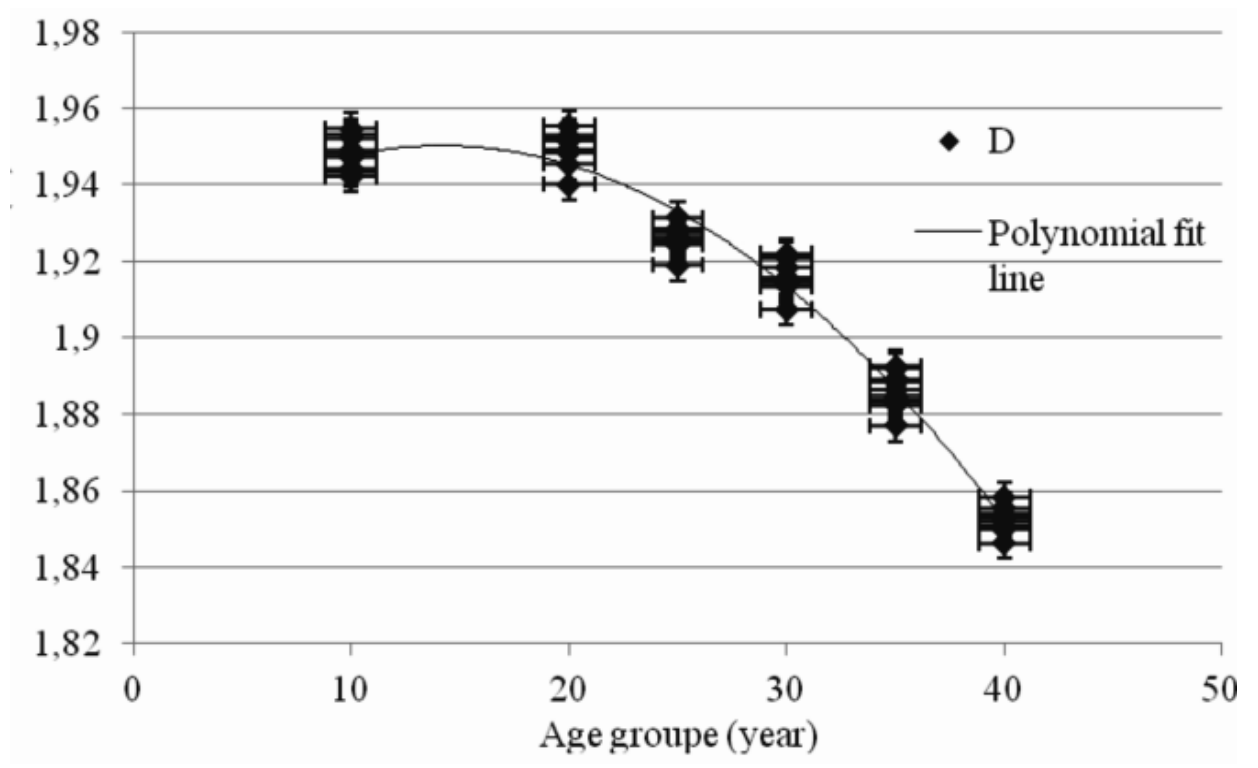

Fig. 2. Particular distribution of the values of fractal dimensions (D) in relation to the age of plum trees, Gras variety 


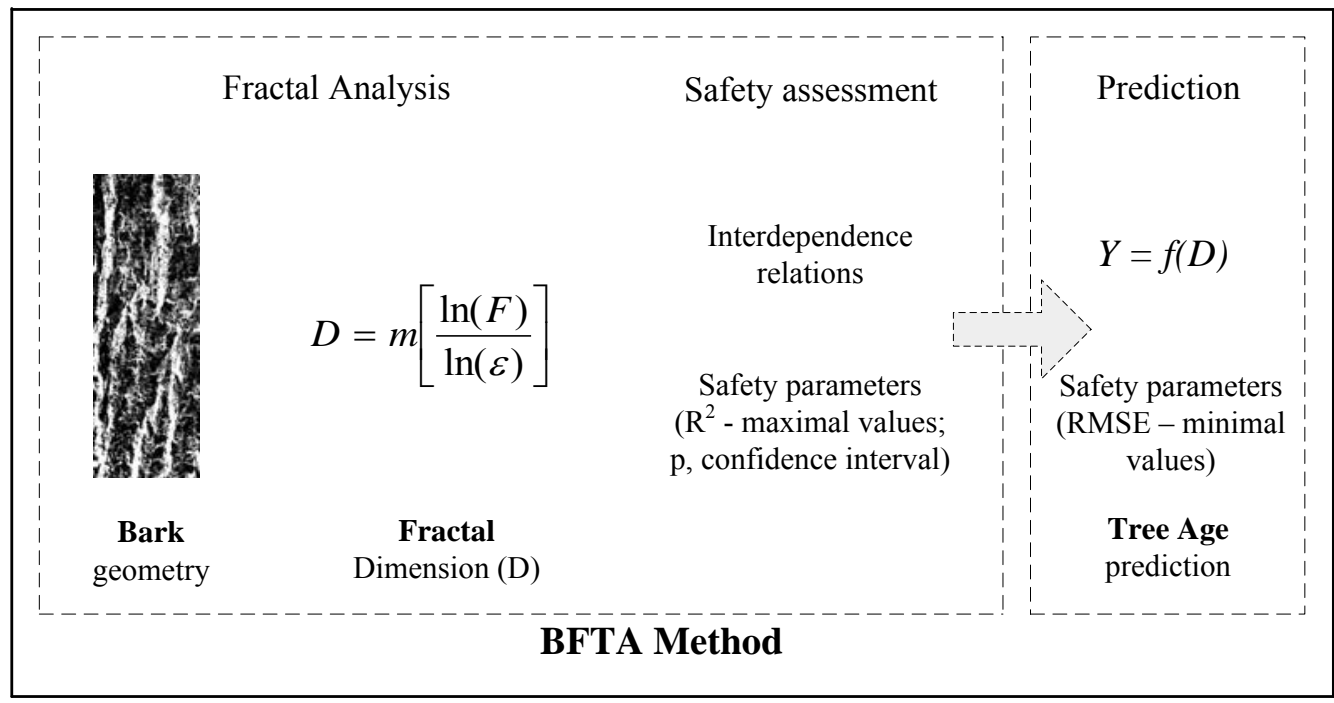

Fig. 3. Bark Fractal Tree Age Method for predicting the age of trees in relation to D

Tab. 3. Mean error and certainty degree of predicting the ages of trees based on fractal dimensions (D)

\begin{tabular}{ccccc}
\hline \multirow{2}{*}{ Age group } & \multicolumn{2}{c}{ Age prediction $Y_{10-40}$} & \multicolumn{2}{c}{ Age prediction $Y_{20-40}$} \\
\cline { 2 - 5 } & Mean error & RMSE & Mean error & RMSE \\
\hline 40 & 1 & 0.8822 & 0 & 0.3865 \\
\hline 35 & 1 & 1.5182 & 0 & 0.7409 \\
\hline 30 & 2 & 1.7824 & 1 & 1.4578 \\
\hline 25 & 4 & 3.8724 & 1 & 1.3441 \\
\hline 20 & 4 & 4.3624 & 0 & 1.6363 \\
\hline 10 & 7 & 7.0461 & - & - \\
\hline
\end{tabular}

Based on the BFTA Method, the present paper assessed the possibility to predict the age of plum trees in relation to the values of the fractal dimensions obtained from the fractal analysis of the bark.

In the interval represented by age groups from 10 to 40 years old, the prediction as possible with medium certainty degree $\left(\mathrm{R}^{2}=0.739 ; \mathrm{p}<0.01\right)$, equation (7). When the interval was limited to the age groups from 20 to 40 years old, the degree of certainty was increased $\left(R^{2}=0.971 ; p<0.01\right)$, equation (8).

$Y_{10-40}=-773.9 x^{2}+2691.4 x-2289.3$

$Y_{20-40}=-801.44 x^{2}+2844.4 x-2478.8$

where: $Y_{10-40}$ - ages of trees in the interval between 10 and 40 years old;
$Y_{20-40}$ - ages of trees in the interval between 20 and 40 years old;

$x$ - fractal dimension (D).

In order to assess the prediction certainty and the accuracy degree of the BFTA Method, the individual values were calculated of the errors for the particular predicted cases. The values of the certainty parameter RMSE were also calculated, equation (9), the data being presented in Table 3.

$$
R M S E=\sqrt{\frac{1}{n} \sum_{j=1}^{n}\left(y_{j}-\widehat{y}_{j}\right)^{2}}
$$

In a larger interval of age groups, the model obtained gave lower certainty prediction, especially with young trees, where the variation coefficient showed higher dynamics in trunk geometry as 
well. The more stable trunk geometry of older trees facilitated high certainty in predicting the age, equation (8), with RMSE values being lowest in 35 to 40 years old groups.

For high degree of certainty in predicting the age of trees, the BFTA Method involves an analysis of fractal properties (D) correlated with the age of trees for every species and variety, on a large number of individuals (trees). In addition, studying subclasses of individuals within the same species/variety (in relation to the exploitation system, technology, etc, factors that can generate particularities of the geometric structure of bark for different age categories) is necessary for obtaining prediction models with high degree of certainty.

Trunk bark was studied through imaging and fractal methods in other studies, which aimed at developing models and methods for the identification of trees based on the geometric properties of their bark. Huang et al. (2006) communicated the results of their studies on bark classification based on textural features using Artificial Neural Networks (ANN). They investigated the relation between Artificial Neural Network (ANN) topologies and bark classification accuracy. The experimental results they obtained show that this new approach can automatically identify plant categories and the classification accuracy of the new method is better than that of the method using the nearest neighbor classifier.
Based on their studies, Fiel and Sablatnig, (2011) proposed a method of automatic identification of tree species based on images of their trunk bark, leaves and needles. They created a database with 1182 images in 11 tree classes. In comparison with the accuracy of tree recognition based on bark images ( 9 images per class and 99 images in total) made $56.6 \%$ by a biologist and $77.8 \%$ by a forest ranger, the proposed method (a Support Vector Machine with radial basis function (RBF) kernel) made it possible to identify trees with $64.2 \%$ and $69.7 \%$ accuracy for training sets with 15 and 30 images per class (where available).

Sulc and Matas (2013) proposed a novel method for tree bark identification by Support Vector Machines (SVM) classification of featuremapped multi-scale descriptors formed by concatenated histograms of Local Binary Patterns (LBPs). Using the standard 15 training examples per class, the proposed method achieves a recognition rate of $82.5 \%$ and significantly outperforms both the state-of-the-art automatic recognition rate of $64.2 \%$ and human experts with recognition rates of $56.6 \%$ and $77.8 \%$.

PCA analysis of experimental data on the fractal dimensions of the geometric structure of tree trunks and their age facilitated a classification of variants in relation to the two parameters, Figure 4 . The two extreme age classes (10 and 50 respectively) were placed on independent

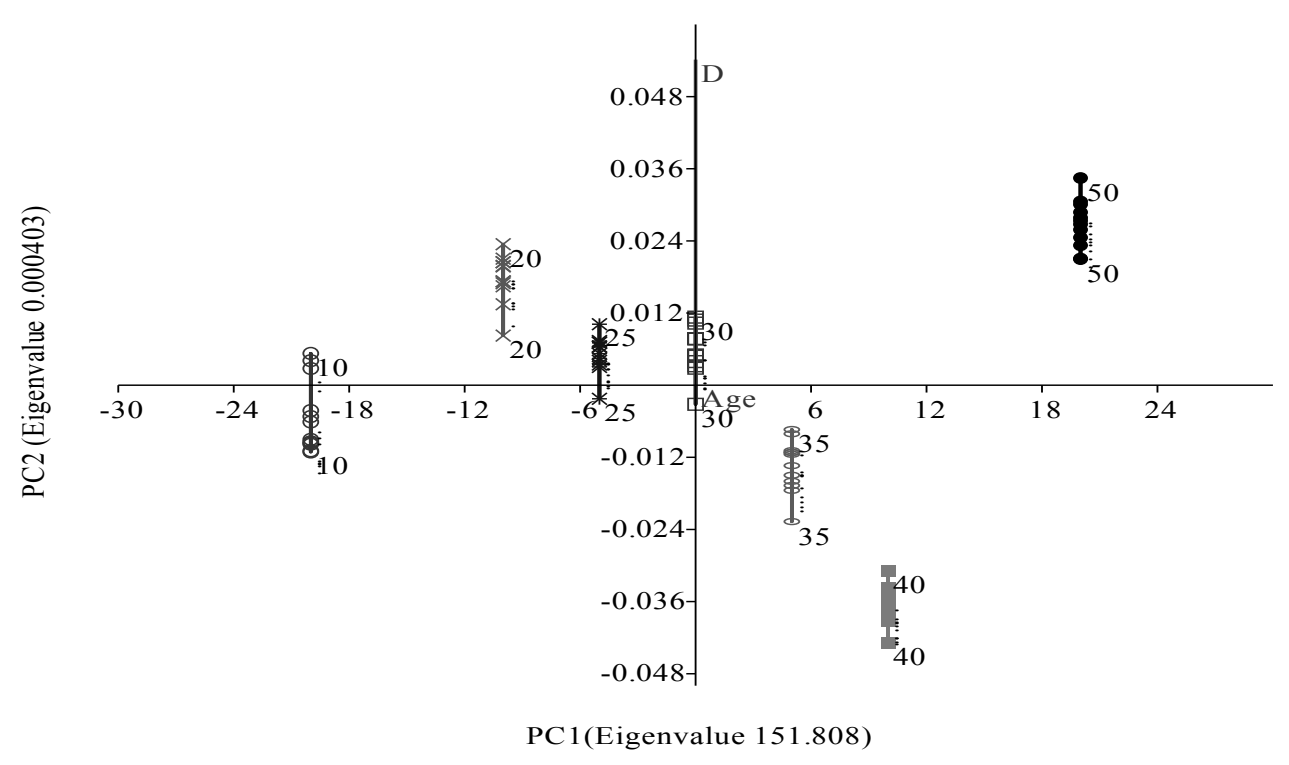

Fig. 4. PCA analysis; variant distribution in relation to the age of the trees and the fractal dimension 
positions. The other variants were grouped in two groups based on affinities: one group made of age classes 20-25-30 and the other composed of age classes 35-40.

Multivariate analysis facilitated the orientation and grouping of variants based on Euclidian distances in two large clusters, in relation to the age of trees and the values of the corresponding fractal dimensions (D). One cluster included the variants in the age groups from 10 to 30 years, and the second included the variants in the age groups from 35 to 50 years, Figure 5. Dendrogram segmentation allowed a finer analysis in what the positioning of variants is concerned. Thus, it became clear that the variants from extreme ages, the youngest (10 years) and oldest (50 years) were placed separately. The other variants were grouped based on affinity: a subcluster including the variants from age groups 20, 25 and 30 years old, with similar values of fractal dimensions, and a second subcluster, containing the variants from groups 30 and 40 years old. According to the cophenetic coefficient with the value 0.766 , the results present high certainty.

\section{CONCLUSIONS}

Fractal analysis focused on the variation in trunk bark geometry of plum, Gras variety, in relation to the ages of trees. A second degree polynomial function described the dependence relation between fractal values (D) and the ages of trees, with high degree of significance and certainty.

Based on the fractal values of bark geometry, it was possible to predict with high certainty the ages of trees. Based on prediction errors calculated and on the RMSE parameter, prediction of the ages of trees based on fractal values (D) had high certainty degree for older age groups (35 to 50 years) than younger age groups within the interval under study.

The Bark Fractal Tree Age (BFTA) Method proposed facilitates the development of applications for assessment and prediction the age of trees and shrubs through non-destructive methods based on fractal analysis. In the case of cultivated fruit trees, the analysis differentiated on species, varieties and technological particularities is required for increasing the certainty degree of the analysis and prediction.

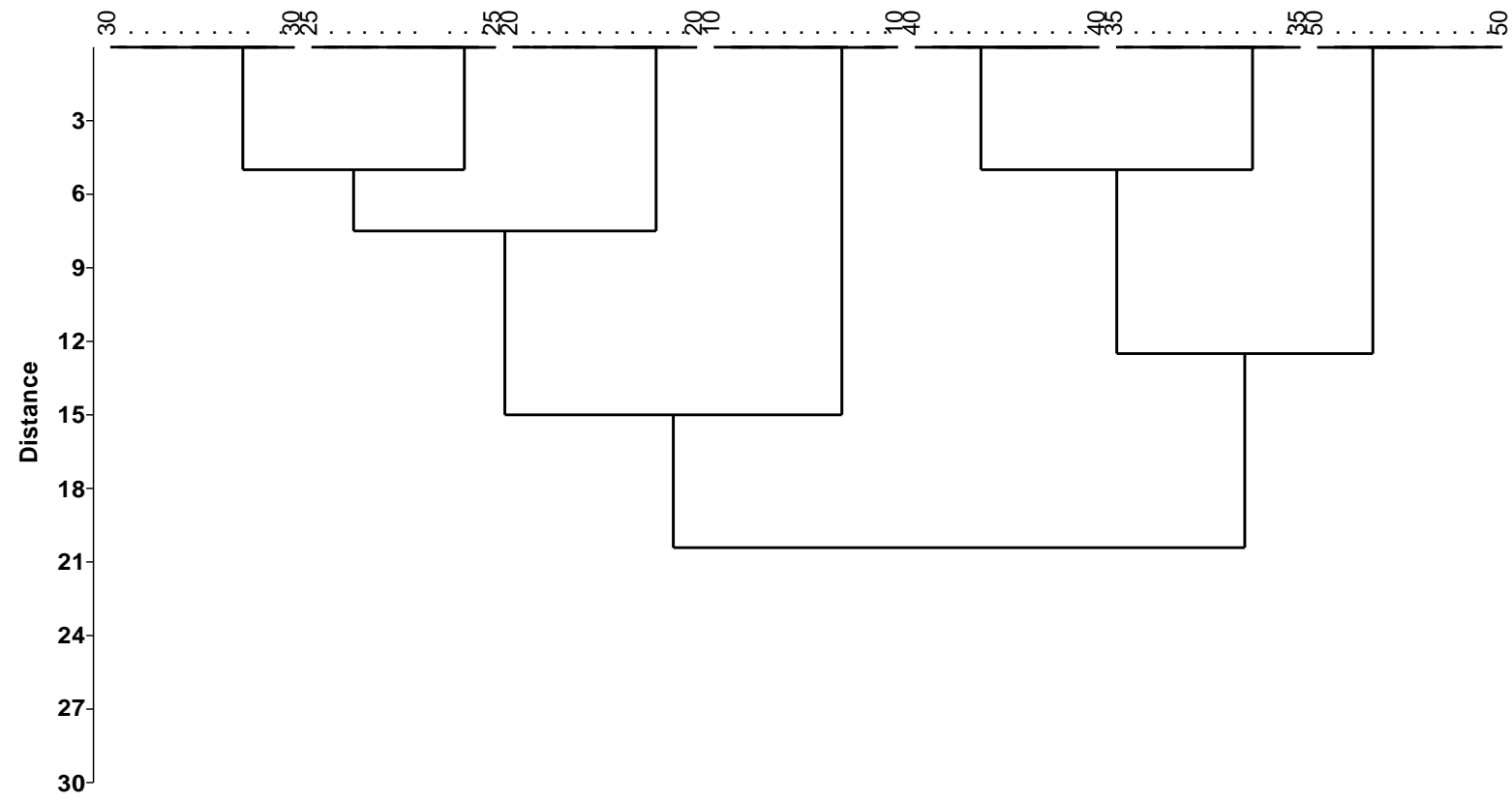

Fig. 5. Dendrogram obtained by cluster analysis ] on the initial data (fractal dimension and age groupe). 


\section{REFERENCES}

1. Backes AR, Bruno OM (2010). Shape classification using complex network and Multi-scale Fractal Dimension. Pattern Recognition Letters 31:44-51.

2. Backes AR, Bruno OM (2013). Texture analysis using volume-radius fractal dimension, Applied Mathematics and Computation 219:5870-5875.

3. Bruno OM, de Oliveira Plotze R, Falvo M, de Castro $M$ (2008). Fractal dimension applied to plant identification, Information Sciences. 178:2722-2733.

4. Bour O, Davy P (1997). Connectivity of random fault networks following a power law fault length distribution. Water Resources Research 33(7):1567-1583.

5. Bour O, Davy P, Darcel C, Odling N (2002). A statistical scaling model for fracture network geometry, with validation on a multiscale mapping of a joint network (Hornelen Basin, Norway). Journal of Geophysical Research 107(B6): 2113.

6. Buchníček M, Nežádal M, Zmeškal O (2000). Numeric Calculation of Fractal Dimension, $3^{\text {rd }}$ Prediction Coference, Faculty of Technology Zlin, BUT Brno, October 2 - 3:10-15.

7. Chi Z, Houqiang L, Chao W (2003). Plant species recognition based on bark patterns using novel Gabor filter banks, Neural Networks and Signal Processing, 2003. Proceedings of the 2003 International Conference on 2:1035-1038.

8. Cope JS, Corney D, Clark JY, Remagnino P, Wilkin P (2012). Plant species identification using digital morphometrics: A review, Expert Systems with Applications 39:7562-7573.

9. Cortini M, Barton CC (1994). Chaos in geomagnetic reversal records - a comparison between earths magnetic - field data and model disk dynamo data, Journal of Geophysical Research 99 (B9):18021-18034.

10. Culbert PD, Radeloff VC, St-Louis V, Flather CH, Rittenhouse CD, Albright TP, Pidgeon AM (2012). Modeling broad-scale patterns of avian species richness across the Midwestern United States with measures of satellite image texture, Remote Sensing of Environment 118:140-150.

11. Du J, Zhai C-M, Wang Q-P (2013). Recognition of plant leaf image based on fractal dimension features, Neurocomputing 116:150-156.

12. Florindo JB, Bruno OM (2012). Fractal descriptors based on Fourier spectrum applied to texture analysis, Physica A 391:4909-4922.

13. Fiel S, Sablatnig R (2011). Automated identification of tree species from images of the bark, leaves and needles, in Proc. of $16^{\text {th }}$ Computer Vision Winter Workshop, Mitterberg, Austria, 2-4 February: 1-6.

14. Gonçalves WN, Bruno OM (2013). Combining fractal and deterministic walkers for texture analysis and classification. Pattern Recognition 46(11): 2953-2968.

15. Hammer $\varnothing$, Harper DAT, Ryan PD (2001). PAST: Paleontological statistics software package for education and data analysis. Palaeontologia Electronica 4(1):1-9.
16. Hein FJ (1999). Mixed ("Multi”) fractal analysis of granite wash fields/pools and structural lineaments, Peace River Arch area, northwestern Alberta, Canada; a potential approach for use in hydrocarbon exploration. Bulletin of Canadian Petroleum Geology. 47(4):556-572.

17. Hou, X-J., R. Gilmore, G.B. Mindlin, H.G. Solari (1990). An efficient algorithm for fast $\mathrm{O}(\mathrm{N} \ln (\mathrm{N}))$ box counting. Physics Letters A.151(1-2):43-46.

18. Huang Z-K, Zheng C-H, Du J-X, Wan Yy (2006). Bark classification based in textural features using artificial neural networks. Advances in Neural Networks - Lecture Notes in Computer Science 3972:355-360.

19. Liebovitch LS, Toth $T$ (1989). A fast algorithm to determine fractal dimensions by box counting, Physics Letters A 141(8-9):386-390.

20. MacFarlane DW, Kuyah S, Mulia R, Dietz J, Muthuri C, Van Noordwijk M (2014). Evaluating a non-destructive method for calibrating tree biomass equations derived from tree branching architecture. Trees 28(3):807-817.

21. Mandelbrot BB (1983). The Fractal Geometry of Nature Revised and Enlarged Edition, W.H. Freeman and Co., New York, $495 \mathrm{pp}$.

22. Nežádal M, Zmeškal O, Buchníček M (2001). The BoxCounting: Critical Study, $4^{\text {th }}$ Prediction Conference, Institute of Information Technologies, Faculty of Technology, Tomas Bata University in Zlin October 25 26:18.

23. Othmani A, Piboule A, Dalmau O, Lomenie N, Mokrani S, Voon LFCLY (2014). Tree species classification based on 3D bark texture analysis, Image and Video Technology, Lecture Notes in Computer Science 8333:279-289.

24. Sulc M, Matas J (2013). Kernel-mapped histograms of multi-scale LBPs for tree bark recognition, Image and Vision Computing New Zealand (IVCNZ), 2013 28th International Conference of, 27-29 Nov::82-87.

25. Thorarinsson F, Magnusson SG (1990). Bouguer density determination by fractal analysis. Geophysics 55(7):932935.

26. Turcotte DL (1992). Fractals and Chaos in Geology and Geophysics. Cambridge University Press, Cambridge 221 pp.

27. Whitmore TC (1962). Studies in systematic bark morphology. New Phytologist 61(2):191-207.

28. Xu Y, Huang S, Ji H, Fernmüller C (2012). Scale-space texture description on SIFT-like textons, Computer Vision and Image Understanding 116(9):999-1013.

29. Zhao Y, Jia W, Hu R-X, Min H (2013). Completed robust local binary pattern for texture classification, Neurocomputing 106:68-76.

30. Zmeškal O, Veselý M, Nežádal M, Buchníček M (2001). Fractal analysis of image structure, HarFA - Harmonic and Fractal Image Analysis 3 - 5. 